\title{
Node Interaction in 802.11 Based Networks with Boolean Interference
}

\author{
Peter van de Ven \\ Eindhoven University of Technology \\ Department of Mathematics and Computer Science \\ Den Dolech 2, 5612 AZ \\ Eindhoven, The Netherlands
}

\author{
Dee Denteneer \\ Philips Research \\ Prof. Holstlaan 4, 5656 AA \\ Eindhoven, The Netherlands
}

\begin{abstract}
Wireless local area networks (WLANs) have been extensively studied over the past several years. So far, research has primarily focussed on scenarios where all nodes in the network can hear each other. The present paper discusses interaction between nodes in a more complex setting, where nodes may only hear parts of the network. The analysis relies on a Markov process that keeps track of the activity of nodes. Return times and hitting probabilities in this process are related to interaction phenomena in the wireless network and examples are given to illustrate how these can be used to predict network performance. The approximations are validated through simulations.
\end{abstract}

\section{INTRODUCTION}

Wireless networks have gone through tremendous developments in the last few decades. One of these advances is the emergence of multi-hop schemes, where packets can travel through intermediate nodes towards their destination. Typically the topology of these types of networks is such that nodes can only sense the activity of a subset of other nodes, which greatly impacts the interaction between nodes. This is even more true for a network using a distributed medium access protocol such as 802.11.

The 802.11 protocol is a variant of Carrier-Sense Multiple Access (CSMA) [1], which can in turn be seen as an extension of the well-known ALOHA protocol [2]. Nodes using the latter protocol access the medium after a random backoff period, regardless of the behaviour of surrounding nodes. Because of the nature of wireless communication, when a node receives multiple strong signals at the same time it might not be able to understand any. So, a natural extension to the ALOHA protocol is then to let a node sense for the activity of surrounding nodes, as CSMA dictates. This greatly reduces the likelihood of collisions. The 802.11 protocol further specifies CSMA by for example adding the binary exponential backoff scheme and by defining interframe spaces. For a detailed description of 802.11 , see for example [3].

Recently some studies have emerged that among others consider node interaction in a multi-hop setting. Wang and Kar [4] use a Markov process based approach to analyze multi-hop networks in saturated conditions. Medepalli and Tobagi [5] investigate an unsaturated network using a mean-value analysis. Garetto et al. [6] take a more elaborate approach, employing separate models to analyze collisions and node behaviour. The authors mention the possibility of extending their analysis to unsaturated networks, but do not go into detail.

In the current paper we present an analysis of the interaction between nodes where each has a fixed offered load, independent of surrounding nodes. While we allow for general routes, we do not explicitly keep track of traffic moving between nodes. The approach is based on the framework Boorstyn and Kershenbaum [7] provide for analyzing packet radio networks, in which they use a Markov process that describes the activity of the nodes. We extend this analysis by using return times and hitting probabilities in the Markov process to capture node interaction.

Our approach differs from the other analyses mentioned in that it is more detailed and accommodates a broader framework that allows for unsaturated networks as well as more involved traffic patterns and unsynchronized nodes. This framework distinguishes a number of models, each describing a different part of the network: the backoff mechanism, node interaction, the time between successive transmissions and network traffic. Each model is then used to input information into the others. For example, information from the node interaction is used to determine the average time between two successful transmissions. This can in turn be used 
in the network model to determine the throughput. See Van de Ven [8] for more details.

The remainder of this paper is structured as follows. Section II provides an outline of the model used for the analysis performed in Section III. In Section IV the approximations developed are compared to simulations, and in Section V we work out an example of how the analysis can be applied to study collisions. Finally, Section VI lists some conclusions. Due to page constraints, only a few proofs are presented in their entirety, while the others are outlined.

\section{MODEL OUTLINE}

We consider a 802.11 based wireless network consisting of a set of nodes $\mathcal{N}$. Traffic flow from one node to another is represented by a link. Let $\mathcal{L}$ denote the set of all links and let $h=\langle a, b\rangle \in \mathcal{L}, a, b \in \mathcal{N}, a \neq b$, then we refer to $a$ as the source of link $h$, and to $b$ as its destination. We make the following simplifying assumptions concerning all links $h \in \mathcal{L}$.

- Whenever link $h$ is transmitting, a set $C_{h}$ of links surrounding $h$ is silenced, independent of other activity in the network;

- similarly, we define a set $I_{h}$ of links such that a transmission of link $h$ fails if and only if one or more links in $I_{h}$ are active.

The latter is called boolean interference, as a transmission over a link in $I_{h}$ always collides with $h$, while links outside of this set never do. Moreover, the assumption also implies a zero-capture collision model, where a transmission fails when a nearby transmission takes place, independent of the progress of the transmission. This is different from perfect capture, where a transmission only fails when a link from $I_{h}$ is active when $h$ activates. Although this model is a simplification, it is fairly realistic, while allowing for tractability. The sets $I_{h}$ and $C_{h}$ can overlap, but neither need to be contained in the other.

To simplify the analysis we assume $k \in C_{h} \Leftrightarrow h \in$ $C_{k}$, and we say that $h$ and $k$ are neighbours when this is true. We also require $h \notin I_{h}, C_{h}$, as link $h$ cannot interfere with, or silence itself. For convenience, we introduce $C_{h}^{+}=C_{h} \cup\{h\}, C_{A}=\bigcup_{h \in A} C_{h}$ and $C_{A}^{+}=C_{A} \cup A, A \subseteq \mathcal{L}$.

At any point in time, a link $h \in \mathcal{L}$ can be either active or inactive, where the former corresponds to a transmission taking place over the link. To facilitate the analysis we assume that during an entire period of activity of a link, its source node is transmitting. This is a slight simplification of the exchange of data and acknowledgements, and the interframe spaces defined in 802.11

We distinguish two types of inactive links: blocked and unblocked. An inactive link $h$ is unblocked when all links in $C_{h}$ are inactive, and is blocked otherwise. An unblocked link $h$ activates after an exponential time with rate $\alpha_{h}$, and the time until an active $h$ link deactivates is exponentially distributed with parameter $\mu_{h}$. So a link only activates when it is unblocked. Here we implicitly assume that the transmission time is exponential, failed transmissions take as long as successful ones and that the time between events of the superposition of the backoff and packet arrival process has an exponential distribution.

This model can be traced back to the dining philosophers' problem introduced by Dijkstra [9], where it is used to study resource sharing in computer networks. It is also similar to models used in some other contexts. This is illustrated by for example Kershenbaum [7] and Tobagi and Brázio [10] for wireless networks, and by Yemini [11] for statistical mechanics.

\section{INTERACTION ANALYSIS}

Let $\{X(t), t \geq 0\}$ denote the set of active links at time $t$. As the time until activation and deactivation of links is exponentially distributed, $X(t)$ is a Markov process. Let $S=\left\{D \subseteq \mathcal{L} \mid C_{D} \cap D=\emptyset\right\}$ be the set of all subsets of links such that none are neighbours, so $S$ is the state space of $X(t)$.

The transition rates of the process are as follows

$$
q(D, E)= \begin{cases}\alpha_{h}, & E=D \cup\{h\}, D \in S, \\ & h \in \mathcal{L} \backslash C_{D}^{+}, \\ \mu_{h}, & E=D \backslash\{h\}, D \in S, h \in D, \\ 0, & \text { otherwise. }\end{cases}
$$

Here we use that $\left\{k \in \mathcal{L} \mid C_{k}^{+} \cap D=\emptyset\right\}=\mathcal{L} \backslash C_{D}^{+}$. Let $P(\cdot)$ denote the limiting distribution of $X(t)$, which can be determined from the global balance equations. For each $D \in S$ :

$$
\begin{aligned}
& P(D)\left(\sum_{h \in D} \mu_{h}+\sum_{k \in \mathcal{L} \backslash C_{D}^{+}} \alpha_{k}\right) \\
& =\sum_{h \in D} P(D \backslash\{h\}) \alpha_{h}+\sum_{k \in \mathcal{L} \backslash C_{D}^{+}} P(D \cup\{k\}) \mu_{k} .
\end{aligned}
$$

By substitution it can easily be shown that

$$
P(D)=P(\emptyset)\left(\prod_{h \in D} g_{h}\right),
$$


where $g_{h}=\alpha_{h} / \mu_{h}$ and $P(\emptyset)=\sum_{D \in S} \prod_{k \in D} g_{k}$, the normalizing constant. So the limiting distribution has a product form. Using the results from Kelly [12, Theorem 1.3], it follows that the process is reversible.

\section{A. Preliminaries}

In this section we introduce additional notation and discuss some preliminaries needed for the analysis in Section III-B. Let $h \in \mathcal{L}, A \subseteq \mathcal{L}$ and define:

1) $\mathcal{A}(h) \subset S$, the set of states where link $h$ is active, $\mathcal{A}(h)=\{D \in S \mid h \in D\}$

2) $\mathcal{B}(h) \subset S$, the set of states where link $h$ is blocked, $\mathcal{B}(h)=\left\{D \in S \mid h \notin D, D \cap C_{h} \neq \emptyset\right\}$

3) $\mathcal{U}(h) \subset S$, the set of states where link $h$ is unblocked, $\mathcal{U}(h)=\left\{D \in S \mid D \cap C_{h}^{+}=\emptyset\right\} ;$

4) $\mathcal{A}(A) \subset S$, the set of states where at least one link in $A$ is active, $\mathcal{A}(A)=\{D \in S \mid D \cap A \neq \emptyset\}$;

Let $H \subseteq S$ and define $P(H)=\sum_{D \in H} P(D), H^{c}=$ $S \backslash H, P_{H}(D)=P(D) / \sum_{E \in H} P(E), D \in H$.

We now introduce the hitting time and return time on $H$.

Definition 1. (Hitting time) The hitting time $T_{H}$ of a subset $H \subseteq S$ is the time it takes for $X(t)$ to reach $H$, starting from time $t=0$ :

$$
T_{H}=\min \{t \geq 0 \mid X(t) \in H\} .
$$

Similarly, we can define the return time.

Definition 2. (Return time) The return time $T_{H}^{+}$on a subset $H \subseteq S$ is the time it takes for $X(t)$ to return to $H$, starting from time $t=0$ :

$$
T_{H}^{+}=\min \left\{t \geq T_{H^{c}} \mid X(t) \in H\right\} .
$$

Lemma 1. (Kac's formula). Let $H \subseteq S$, then

$$
\mathrm{E}\left[T_{H} \mid X(0) \sim \rho_{H}\right]=\frac{P\left(H^{c}\right)}{Q(H)},
$$

where $\rho_{H}$ is the distribution of the process immediately after exiting $H$ and $Q(H)$ the average rate out of $H$ :

$$
\begin{aligned}
\rho_{H}(E) & =\sum_{D \in H} \frac{P(D)}{Q(H)} q(D, E), \quad E \in H^{c}, \\
Q(H) & =\sum_{D \in H} \sum_{E \in H^{c}} P(D) q(D, E) .
\end{aligned}
$$

The next lemma shows that when the process is in steady state, the time until a link activates is approximately exponentially distributed. In order to show this, we need a slightly alternate view of the activation process of link $h$, and introduce the concept of activation attempts. Instead of links activating after an exponential time when they are unblocked, we introduce a constant Poisson process of activation attempts with rate $\alpha_{h}$. When such an activation attempts occurs, and link $h$ is unblocked, this link activates. Because the time between successive activation attempts is exponentially distributed, these two systems are equivalent.

Lemma 2. Let $A, B \subseteq \mathcal{L}$, such that $A \cap B=\emptyset, A \cap C_{A}=$ $\emptyset$ and take $H=\bigcap_{h \in A} \mathcal{A}(h) \cap \mathcal{A}^{c}(B)$. Let $\widetilde{T}_{\mathcal{A}(k)}$ denote the approximation of $T_{\mathcal{A}(k)}$ under the assumption that successive activation attempts see the system in steady state. Then

$$
\begin{aligned}
& \mathbb{P}\left(\widetilde{T}_{\mathcal{A}(k)}>t \mid X(0) \sim P_{H}, T_{H^{c}}^{+} \geq T_{\mathcal{A}(k)}\right) \\
& =\exp \left(-t \alpha_{k} P_{H}(\mathcal{U}(k))\right), \quad k \in \mathcal{L} \backslash\left(C_{A}^{+} \cup B\right) .
\end{aligned}
$$

So we fix the set of links $A$ and $B$ to be active and inactive respectively, and approximate the time until link $k$ activates. The result can be explained by the observation that, under the aforementioned assumption, the time until activation is in fact a geometric sum of independent exponentially distributed random variables.

We now introduce the sum of products.

Definition 3. (Sum of products) The sum of products (SP) of a set $A \subseteq \mathcal{L}$ is the non-normalized sum of the limiting probabilities over all possible states containing just links in A:

$$
\mathrm{SP}(A)=\sum_{\substack{D \subseteq A \\ D \in S}} \prod_{h \in D} g_{h} .
$$

The following properties of the SP can easily be proven by writing out its definition.

Lemma 3. Let $A_{1}, A_{2}, A \subseteq \mathcal{L}$, and let $A_{1}$ and $A_{2}$ be such that $C_{A_{1}}^{+} \cap A_{2}=\emptyset$, then

1) $\mathrm{SP}\left(A_{1} \cup A_{2}\right)=\mathrm{SP}\left(A_{1}\right) \mathrm{SP}\left(A_{2}\right)$,

2) $\mathrm{SP}(A)=\mathrm{SP}(A \backslash B)+\sum_{\substack{D \subseteq B, D \in S \\ D \neq \emptyset}}(\mathrm{SP}(A \backslash(B \cup$ $\left.\left.\left.C_{D}\right)\right) \prod_{h \in D} g_{h}\right), \quad B \subseteq A$,

3) $\mathrm{SP}(A)=\operatorname{SP}(A \backslash\{h\})+g_{h} \operatorname{SP}\left(A \backslash C_{h}^{+}\right), \quad h \in A$.

\section{B. Interaction analysis}

We now use the preliminaries from Section III-A to derive some properties of the Markov process under 
consideration. These properties are related to link interaction. Let $h \in \mathcal{L}$ be a link, then we are interested in the following quantities.

1) $\mathrm{E}[B(h)]$, the average duration of a blocked period of link $h$;

2) $p_{0}(h)=\mathbb{P}\left(X(0) \in \mathcal{A}\left(I_{h}\right) \mid X(0) \sim \rho_{\mathcal{A}^{c}(h)}\right)$, the probability that any link in $I_{h}$ is active when link $h$ activates;

3) $p_{1}(h)=\mathbb{P}\left(T_{\mathcal{A}\left(I_{h}\right)}<T_{\mathcal{A}^{c}(h)} \mid X(0) \sim\right.$ $\left.\rho_{\mathcal{A}^{c}(h)}, X(0) \notin \mathcal{A}\left(I_{h}\right)\right)$, the probability that a link in $I_{h}$ activates before link $h$ deactivates, given that $h$ just activated and no link in $I_{h}$ is active;

4) $p_{b}(h)=\mathbb{P}\left(T_{\mathcal{B}(h)}<T_{\mathcal{A}(h)} \mid X(0) \sim \rho_{\mathcal{U}^{c}(h)}\right)$ the probability that link $h$ becomes blocked before it activates, given that it just became unblocked.

Here $\mathrm{E}[B(h)]$ and $p_{0}(h)$ will be determined exactly, while $p_{1}(h)$ and $p_{b}(h)$ will be approximated. As mentioned before, these quantities can be used in a broader context to determine the time between successive transmissions in [8]. This is done by constructing a Markov process with four states representing the state of a link: blocked, unblocked, collision and successful transmission. We are interested in the return time from the state representing a successful transmission to itself, as this is the time between two successful transmissions. The rate at which each state is exited, as well as the transmission probabilities needed in [8] depend on the average blocked time, collision probabilities etc., which are exactly the quantities that we will determine in this section.

Because of the difficult structure of the process, it seems impossible to derive the quantities needed directly. Instead, we construct approximations using the description of the process rather than its structure.

\section{Lemma 4.}

$$
\mathrm{E}[B(h)]=\frac{1-P(\emptyset) \operatorname{SP}\left(\mathcal{L} \backslash C_{h}^{+}\right)\left(1+g_{h}\right)}{P(\emptyset) \sum_{k \in C_{h}} \alpha_{k} \operatorname{SP}\left(\mathcal{L} \backslash\left(C_{h}^{+} \cup C_{k}^{+}\right)\right)} .
$$

This result can easily be obtained using Kac's formula.

\section{Lemma 5.}

$$
p_{0}(h)=1-\frac{\operatorname{SP}\left(\mathcal{L} \backslash\left(C_{h}^{+} \cup I_{h}\right)\right)}{\operatorname{SP}\left(\mathcal{L} \backslash C_{h}^{+}\right)} .
$$

Again, we can use Kac's formula to write out the definition of $p_{0}(h)$.

Let $\widetilde{p_{1}}(h)$ be the approximation to $p_{1}(h)$ under the assumption that (i) the links in $I_{h}$ activate independently and (ii) the distribution of the process just after link $h$ activates given that $I_{h}$ is deactivated is $P_{\mathcal{A}(h) \cap \mathcal{A}^{c}\left(I_{h}\right)}$.

\section{Lemma 6.}

$$
\widetilde{p_{1}}(h)=1-\frac{\mu_{h}}{\mu_{h}+\sum_{k \in I_{h}} \alpha_{k} \frac{\operatorname{SP}\left(\mathcal{L} \backslash\left(C_{h}^{+} \cup C_{k}^{+} \cup I_{h}\right)\right)}{\operatorname{SP}\left(\mathcal{L} \backslash\left(C_{h}^{+} \cup I_{h}\right)\right)}} .
$$

Proof: Using the assumption concerning the initial distribution of the process:

$$
\widetilde{p_{1}}(h)=\mathbb{P}\left(T_{\mathcal{A}\left(I_{h}\right)}<T_{\mathcal{A}^{c}(h)} \mid X(0) \sim P_{\mathcal{A}(h) \cap \mathcal{A}^{c}\left(I_{h}\right)}\right) .
$$

Denoting

$R(t)=\mathbb{P}\left(T_{\mathcal{A}\left(I_{h}\right)}<t \mid X(0) \sim P_{\mathcal{A}(h) \cap \mathcal{A}^{c}\left(I_{h}\right)}, T_{\mathcal{A}^{c}(h)}=t\right)$, and conditioning on $T_{\mathcal{A}^{c}(h)}$ yields

$$
\begin{aligned}
\widetilde{p_{1}}(h) & =\int_{t=0}^{\infty} R(t) \mathrm{d} \mathbb{P}\left(T_{\mathcal{A}^{c}(h)} \leq t\right) \\
& =\int_{t=0}^{\infty} \mu_{h} \mathrm{e}^{-\mu_{h} t}(1-(1-R(t))) \mathrm{d} t .
\end{aligned}
$$

Now, using the independence assumption and Lemma 2:

$$
\begin{aligned}
& \mathbb{P}\left(T_{\mathcal{A}(k)} \geq t \mid X(0) \sim P_{\mathcal{A}(h) \cap \mathcal{A}^{c}\left(I_{h}\right)}, T_{\mathcal{A}^{c}(h)}=t\right) \\
& =\exp \left(-\alpha_{k} t P\left(\mathcal{U}(k) \mid \mathcal{A}(h) \cap \mathcal{A}^{c}\left(I_{h}\right)\right)\right) .
\end{aligned}
$$

Substituting this into Equation (1) yields

$$
\begin{aligned}
\widetilde{p_{1}}(h) & =\int_{t=0}^{\infty} \mu_{h} \mathrm{e}^{-\mu_{h} t} \\
& \left(1-\prod_{k \in I_{h}} \exp \left(-\alpha_{k} t P\left(\mathcal{U}(k) \mid \mathcal{A}(h) \cap \mathcal{A}^{c}\left(I_{h}\right)\right)\right)\right) \mathrm{d} t \\
& =1-\mu_{h} \int_{t=0}^{\infty} \exp \\
& \left(-t\left(\mu_{h}+\sum_{k \in I_{h}} \alpha_{k} P\left(\mathcal{U}(k) \mid \mathcal{A}(h) \cap \mathcal{A}^{c}\left(I_{h}\right)\right)\right)\right) \mathrm{d} t \\
& =1-\frac{\mu_{h}}{\mu_{h} \sum_{k \in I_{h}} \alpha_{k} P\left(\mathcal{U}(k) \mid \mathcal{A}(h) \cap \mathcal{A}^{c}\left(I_{h}\right)\right)} \\
& =1-\frac{\mu_{h}}{\mu_{h}+\sum_{k \in I_{h}} \alpha_{k} \frac{P\left(\mathcal{U}(k) \cap \mathcal{A}(h) \cap \mathcal{A}^{c}\left(I_{h}\right)\right)}{P\left(\mathcal{A}(h) \cap \mathcal{A}^{c}\left(I_{h}\right)\right)}} \\
& =1-\frac{\mu_{h}}{\mu_{h}+\sum_{k \in I_{h}} \alpha_{k} \frac{P(\emptyset) \operatorname{SP}\left(\mathcal{L} \backslash\left(C_{h}^{+} \cup C_{k}^{+} \cup I_{h}\right)\right) g_{h}}{P(\emptyset) \operatorname{SP}\left(\mathcal{L} \backslash\left(C_{h}^{+} \cup I_{h}\right)\right) g_{h}}} \\
& =1-\frac{\mu_{h}}{\mu_{h}+\sum_{k \in I_{h}} \alpha_{k} \frac{\operatorname{SP}\left(\mathcal{L} \backslash\left(C_{h}^{+} \cup C_{k}^{+} \cup I_{h}\right)\right)}{\operatorname{SP}\left(\mathcal{L} \backslash\left(C_{h}^{+} \cup I_{h}\right)\right)}}
\end{aligned}
$$


Note that when $I_{h}=\{k\}$ and $\mu_{h}=\mu_{k}$,

$$
\begin{aligned}
\widetilde{p_{1}}(h) & =1-\frac{\mu_{h}}{\mu_{h}+\sum_{k \in I_{h}} \alpha_{k} \frac{\operatorname{SP}\left(\mathcal{L} \backslash\left(C_{h}^{+} \cup C_{k}^{+} \cup I_{h}\right)\right)}{\operatorname{SP}\left(\mathcal{L} \backslash\left(C_{h}^{+} \cup I_{h}\right)\right)}} \\
& =1-\frac{\operatorname{SP}\left(\mathcal{L} \backslash\left(C_{h}^{+} \cup\{k\}\right)\right)}{\operatorname{SP}\left(\mathcal{L} \backslash\left(C_{h}^{+} \cup\{k\}\right)\right)+g_{k} \operatorname{SP}\left(\mathcal{L} \backslash\left(C_{h}^{+} \cup C_{k}^{+}\right)\right)} \\
& =1-\frac{\operatorname{SP}\left(\mathcal{L} \backslash\left(C_{h}^{+} \cup\{k\}\right)\right)}{\operatorname{SP}\left(\mathcal{L} \backslash C_{h}^{+}\right)} \\
& =p_{0}(h),
\end{aligned}
$$

\begin{tabular}{|c|c|c|c|c|c|}
\hline & $a$ & 1 & 2 & 3 & 4 \\
\hline \multirow{3}{*}{$N=6$} & $\widetilde{p_{1}}\left(h_{a}\right)$ & 0.458 & 0.444 & - & - \\
\hline & $p_{1}\left(h_{a}\right)$ simulation & 0.377 & 0.445 & - & - \\
\hline & $\Delta \%$ & 21.4 & -0.2 & - & - \\
\hline \multirow{3}{*}{$N=7$} & $\widetilde{p_{1}}\left(h_{a}\right)$ & 0.415 & 0.348 & 0.500 & - \\
\hline & $p_{1}\left(h_{a}\right)$ simulation & 0.350 & 0.326 & 0.507 & - \\
\hline & $\Delta \%$ & 18.6 & 6.7 & -1.3 & - \\
\hline \multirow{3}{*}{$N=8$} & $\widetilde{p_{1}}\left(h_{a}\right)$ & 0.468 & 0.296 & 0.417 & 0.166 \\
\hline & $p_{1}\left(h_{a}\right)$ simulation & 0.397 & 0.266 & 0.388 & 0.166 \\
\hline & $\Delta \%$ & 17.9 & 11.3 & 7.2 & 0.0 \\
\hline
\end{tabular}

Table I

THE RELATIVE DIFFERENCES OF $\widetilde{p_{1}}\left(h_{a}\right)$ PER LINK FOR VARIOUS VALUES OF $N$.

where we use Lemma 3.3 in the third step.

We continue to approximate $p_{b}(h)$. Similar to what was done for $p_{1}(h)$, we approximate $p_{b}(h)$ by $\widetilde{p_{b}}(h)$. We calculate $\widetilde{p_{b}}(h)$ as if (i) the links in $C_{h}$ activate independent from each other and (ii) the distribution of the process just after link $h$ becomes unblocked is $P_{\mathcal{U}}(h)$. The derivation of the approximation is very similar to that of Lemma 6

\section{Lemma 7.}

$$
\widetilde{p_{b}}(h)=1-\frac{\alpha_{h}}{\alpha_{h}+\sum_{k \in C_{h}} \alpha_{k} \frac{\operatorname{SP}\left(\mathcal{L} \backslash\left(C_{h}^{+} \cup C_{k}^{+}\right)\right)}{\operatorname{SP}\left(\mathcal{L} \backslash C_{h}^{+}\right)}} .
$$

\section{VALIDATION}

In order to verify the quality of the approximations $\widetilde{p_{1}}$ and $\widetilde{p_{b}}$, we compare them to simulations. We do not simulate the wireless network itself using a simulator such as NS2. Instead, a Java simulation of the model described in Section II is used. We consider a chain topology with $N$ nodes, where a node can hear neighbours two hops away. Nodes transmit packets towards their right neighbour, so collisions occur when the third neighbour on the right starts transmitting. Let $h_{a}$ denote the $a$ th link, between nodes $a, a+1$, then

$$
C_{h_{a}}=\left\{h_{a-2}, h_{a-1}, h_{a+1}, h_{a+2}\right\}, \quad I_{h_{a}}=\left\{h_{a+3}\right\} .
$$

Finally, we use the following parameters:

$$
\begin{aligned}
\alpha_{h_{a}} & =0.2-0.03(a-1), \quad a=1,2, \ldots, N-1, \\
\mu_{h_{a}} & = \begin{cases}0.05, & a \text { odd }, \\
0.1, & a \text { even. }\end{cases}
\end{aligned}
$$

Tables I and II contain the values and relatives differences for $p_{1}$ and $p_{b}$ respectively, for various values of $N$. Because $p_{1}\left(h_{a}\right)=0$ for the rightmost three links, Table I does not contain results for these links. Both tables suggest that the approximation is quite accurate. The accuracy of $\widetilde{p_{1}}\left(h_{a}\right)$ seems to depend on the length of the chain as well as the position of the link. On the other hand, the results for $\widetilde{p_{b}}\left(h_{a}\right)$ are accurate up to the point where they appear to be within the margin of error of the simulation.

\section{EXAMPLE}

Besides being applicable in the broader context of the framework presented in [8], a part of our contribution can be used to extend the analysis presented by Boorstyn et al. [13]. Specifically, the collision probabilities $p_{0}(h)$, $p_{1}(h)$ can be used to soften the unrealistic assumption of perfect capture.

We are interested in the throughput of each link, defined as the fraction of time a link is active working on a successful transmission. Let $\gamma_{p}(h), \gamma_{z}(h)$ denote the throughput in the perfect capture and zero capture model respectively:

$$
\begin{aligned}
& \gamma_{p}(h)=g_{h} \operatorname{SP}\left(\mathcal{L} \backslash C_{h}^{+}\right)\left(1-p_{0}(h)\right), \\
& \gamma_{z}(h)=g_{h} \operatorname{SP}\left(\mathcal{L} \backslash C_{h}^{+}\right)\left(1-p_{0}(h)\right)\left(1-p_{1}(h)\right) .
\end{aligned}
$$

When approximating $p_{1}(h)$ by $\widetilde{p_{1}}(h), \gamma_{z}(h)$ by $\widetilde{\gamma_{z}}(h)$ and considering the chain topology and parameter setting discussed in Section IV, we get the results displayed in Table III. This table shows relaxing the perfect capture assumption has a significant impact on throughput.

\section{CONCLUSion}

We have constructed an approximation for the interaction between links in a 802.11 based wireless network. This is done by interpreting the return times and hitting probabilities in a Markov process tracking the activity of links as interaction phenomena. Comparison to simulations demonstrates that the approximations are quite accurate, at least for linear topologies. 


\begin{tabular}{|c|c|c|c|c|c|c|c|c|}
\hline & $a$ & 1 & 2 & 3 & 4 & 5 & 6 & 7 \\
\hline \multirow{3}{*}{$N=6$} & $\widetilde{p_{b}}\left(h_{a}\right)$ & 0.440 & 0.635 & 0.800 & 0.563 & 0.562 & - & - \\
\hline & $p_{b}\left(h_{a}\right)$ simulation & 0.441 & 0.635 & 0.799 & 0.564 & 0.563 & - & - \\
\hline & $\Delta \%$ & -0.2 & 0.0 & 0.1 & -0.2 & -0.2 & - & - \\
\hline \multirow{3}{*}{$N=7$} & $\widetilde{p_{b}}\left(h_{a}\right)$ & 0.467 & 0.641 & 0.780 & 0.636 & 0.657 & 0.696 & - \\
\hline & $p_{b}\left(h_{a}\right)$ simulation & 0.468 & 0.639 & 0.780 & 0.637 & 0.656 & 0.696 & - \\
\hline & $\Delta \%$ & -0.2 & 0.3 & 0.0 & -0.2 & 0.2 & 0.0 & - \\
\hline \multirow{3}{*}{$N=8$} & $\widetilde{p_{b}}\left(h_{a}\right)$ & 0.461 & 0.653 & 0.779 & 0.585 & 0.684 & 0.729 & 0.771 \\
\hline & $p_{b}\left(h_{a}\right)$ simulation & 0.459 & 0.652 & 0.782 & 0.585 & 0.682 & 0.733 & 0.770 \\
\hline & $\Delta \%$ & 0.4 & 0.2 & -0.3 & 0.0 & 0.3 & -0.5 & 0.1 \\
\hline
\end{tabular}

Table II

THE RELATIVE DIFFERENCES OF $\widetilde{p_{b}}\left(h_{a}\right)$ PER LINK FOR VARIOUS VALUES OF $N$.

\begin{tabular}{ll|lllllll}
\multicolumn{1}{c}{$a$} & 1 & 2 & 3 & 4 & 5 & 6 & 7 \\
\hline \multirow{2}{*}{$N=6$} & $\gamma_{p}\left(h_{a}\right)$ & 0.404 & 0.066 & 0.109 & 0.214 & 0.417 & - & - \\
& $\widetilde{\gamma}_{z}\left(h_{a}\right)$ & 0.219 & 0.037 & 0.109 & 0.214 & 0.417 & - & - \\
\hline \multirow{2}{*}{$N=7$} & $\gamma_{p}\left(h_{a}\right)$ & 0.407 & 0.084 & 0.092 & 0.181 & 0.352 & 0.156 & - \\
& $\widetilde{\gamma}_{z}\left(h_{a}\right)$ & 0.238 & 0.055 & 0.046 & 0.181 & 0.352 & 0.156 & - \\
\hline \multirow{2}{*}{$N=8$} & $\gamma_{p}\left(h_{a}\right)$ & 0.384 & 0.089 & 0.108 & 0.151 & 0.294 & 0.130 & 0.165 \\
& $\widetilde{\gamma}_{z}\left(h_{a}\right)$ & 0.204 & 0.062 & 0.063 & 0.126 & 0.294 & 0.130 & 0.165
\end{tabular}

Table III

THE THROUGHPUT PER LINK WITH PERFECT CAPTURE AND ZERO CAPTURE FOR VARIOUS VALUES OF $N$.

We have also presented two possible applications of the results derived in this paper. The first is a broader framework presented in [8], which provides a detailed yet mathematically tractable analysis of unsaturated 802.11 based networks. Moreover, using part of our contribution it is possible to extend the model by Boorstyn et al. [13] to relax the unrealistic assumption of perfect capture.

\section{ACKNOWLEDGMENT}

We would like to thank Sem Borst for his valuable comments on earlier drafts of this paper.

\section{REFERENCES}

[1] L. Kleinrock and F. Tobagi, "Packet switching in radio channels: part I - carrier sense multiple-access modes and their throughput-delay characteristics," IEEE Transactions on Communications, vol. 23, no. 12, pp. 1400-1416, 1975.

[2] N. Abramson, "The ALOHA system - another alternative for computer communications," in Proc. of AFIPS, 1970, pp. 281285.

[3] 802.11, "IEEE standards for information technology - Telecommunications and information exchange between systems - local and metropolitan area network - specific requirements - Part 11: wireless LAN medium access control (MAC) and physical layer (PHY) specifications," 1999.

[4] X. Wang and K. Kar, "Throughput modelling and fairness issues in CSMA/CA based ad-hoc networks," in Proc. of INFOCOM, 2005, pp. 23-34.
[5] K. Medepalli and F. Tobagi, "Towards performance modeling of IEEE 802.11 based wireless networks: a unified framework and its applications," in Proc. of INFOCOM, 2006.

[6] M. Garetto, T. Salonidis, and E. Knightly, "Modeling perflow throughput and capturing starvation in CSMA multi-hop wireless networks," in Proc. of INFOCOM, 2006.

[7] R. Boorstyn and A. Kershenbaum, "Throughput analysis of multihop packet radio," in Proc. of ICC, 1980, pp. 1361-1366.

[8] P. Van de Ven, "Throughput and delay evaluation of 802.11based multi-hop wireless networks," Master's thesis, Eindhoven University of Technology, 2007.

[9] E. Dijkstra, "The structure of the THE multiprogramming system," Communications of the ACM, vol. 11, no. 5, pp. 341346, 1968.

[10] F. Tobagi and J. Brázio, "Throughput analysis of multihop packet radio networks under various channel access schemes," in Proc. of INFOCOM, 1983, pp. 381-389.

[11] Y. Yemini, "A statistical mechanics of distributed resource sharing mechanisms," in Proc. of INFOCOM, 1983, pp. 531539.

[12] F. Kelly, Reversibility and Stochastic Networks, 1st ed. New York: Wiley, 1979.

[13] R. Boorstyn, A. Kershenbaum, B. Maglaris, and V. Sahin, "Throughput analysis in multihop CSMA packet radio networks," IEEE Transactions on Communications, vol. 35, no. 3 , pp. 267-274, 1987. 